\title{
ELETROMANOMETRIA ESOFÁGICA E pHMETRIA DE 24 HORAS NA AVALIAÇÃO PÓS-OPERATÓRIA DA HIATOPLASTIA E VÁLVULA ANTI-REFLUXO TOTAL LAPAROSCÓPICA
}

\author{
Ricardo PASTORE ${ }^{1}$, Eduardo CREMA ${ }^{1}$, \\ Mariana de Carvalho SILVEIRA ${ }^{1}$, Adriana Ferreira PRESOTO ${ }^{1}$, \\ Fernando Augusto Mardiros HERBELLA ${ }^{2}$ e José Carlos Del GRANDE ${ }^{2}$.
}

RESUMO - Racional - A operação de "short floppy Nissen” tem como objetivo tratar o refluxo gastroesofágico. Avaliações clínicas e endoscópicas de sua eficiência têm sido demonstradas por diferentes autores, porém estudos comparando pHmetria ácida de 24 horas com exames realizados no pré e pós-operatório têm sido pouco freqüentes. Objetivos - Avaliar os resultados da hiatoplastia e válvula anti-refluxo total laparoscópica no tratamento da doença do refluxo gastroesofágico não complicada através de estudo comparativo pré e pós-operatório de aspectos manométricos e pHmétricos. Métodos - Cinqüenta e nove indivíduos com sintomas típicos de refluxo gastroesofágico submetidos a cirurgia de "short floppy Nissen" por laparoscopia foram estudados prospectiva e consecutivamente no período de março de 2002 a agosto de 2003 . Realizou-se no período pré e pós-operatório, em um tempo mínimo de 3 meses, endoscopia digestiva alta, manometria esofágica e pHmetria ácida de 24 horas. Resultados - Dezoito (30,5\%) doentes eram do sexo masculino e 41 (69,5\%) do feminino, com idade média de 43,8 anos. Houve diferença nos seguintes achados manométricos, quando comparados no período pré e pós-operatório: localização do esfíncter inferior do esôfago em relação à borda nasal; extensão do esfíncter inferior do esôfago; pressão de repouso do esfíncter inferior do esôfago; pressão do corpo esofágico. Houve diferença nos seguintes achados pHmétricos quando comparados no período pré e pós-operatório: redução do número de refluxo ácidos totais; número de refluxos ácidos prolongados; número de refluxos ácidos prolongados em decúbito dorsal; número de refluxos ácidos prolongados em posição ereta; fração de tempo de acidificação em minutos; índice de DeMeester. Conclusões - A eletromanometria e a pHmetria esofágicas mostraram melhora em cada um dos seus parâmetros com significância estatística entre os pacientes no período pré e pós-operatório de hiatoplastia e válvula anti-refluxo, demonstrando a eficiência do procedimento operatório quando avaliado por esses exames.

DESCRITORES - Refluxo gastroesofágico. Manometria. Hérnia hiatal. Fundoplicatura. Laparoscopia.

\section{INTRODUÇÃO}

A doença do refluxo gastroesofágico (DRGE) representa grande impacto na qualidade de vida dos indivíduos e as complicações desta enfermidade, especificamente o esôfago de Barrett, tornam importante a caracterização e o tratamento precoce desta afecção ${ }^{(10,14,18)}$.
A cirurgia é opção de tratamento cada vez mais freqüente para a DRGE. Os critérios para sua indicação são principalmente a falha do tratamento clínico e a DRGE complicada $^{(7,9)}$. O tratamento cirúrgico estabeleceu-se definitivamente com o advento do acesso laparoscópico, realizado pela primeira vez por Dallemagne e Geagea em 1991, tendo se popularizado a partir de então ${ }^{(8,17)}$,

${ }^{1}$ Disciplina de Cirurgia do Aparelho Digestivo da Faculdade de Medicina do Triângulo Mineiro, Uberaba, MG; ${ }^{2}$ Disciplina de Cirurgia Gastroenterológica da Universidade Federal de São Paulo - UNIFESP, São Paulo, SP.

Endereço para correspondência: Dr. Ricardo Pastore - Rua Duque de Caxias, 624 - Bairro São Benedito - 38022-180 - Uberaba, MG. 
devido aos seus bons resultados, baixo índice de complicações e mudança para melhor na qualidade de vida ${ }^{(1)}$.

A eletromanometria esofágica permite a identificação do esfíncter inferior do esôfago (EIE), sua extensão, pressão basal e eficácia em relaxar ante o estímulo da deglutição ${ }^{(12)}$. Por possibilitar a mensuração de características anatomofisiológicas do esôfago, aplicando-lhes valores numéricos, tem sido considerada atualmente importante subsídio, não só na prática médica diária, mas principalmente, na pesquisa clínica ${ }^{(3)}$.

Com o desenvolvimento dos dispositivos de monitorização prolongada do $\mathrm{pH}$ intraluminar esofágico, ampliou-se ainda mais o conhecimento fisiopatológico da doença. Constatouse que alguns pacientes apresentavam sintomas sugestivos da DRGE sem, no entanto, haver esofagite identificável pelo exame endoscópico do esôfago. Quando submetidos a monitorização prolongada do $\mathrm{pH}$ intraluminar do esôfago, apresentavam índices de refluxo considerados patológicos, ou seja, em níveis superiores aos encontrados em indivíduos normais. Chegou-se, assim, à concepção atual da DRGE, onde se entende que o refluxo gastroesofágico pode determinar ou não esofagite ${ }^{(15)}$.

A operação de "short floppy Nissen" tem como objetivo tratar o refluxo gastroesofágico. Avaliações clínicas e endoscópicas da eficiência da cirurgia têm sido demonstradas por diferentes autores porém, estudos com a realização de pHmetria ácida de 24 horas e manometria no pós-operatório, têm sido pouco freqüentes, tanto na literatura nacional, quanto mundial.

\section{MATERIAL E MÉTODO}

No período de março de 2002 a agosto de 2003, foram operados consecutivamente 59 indivíduos com sintomas típicos de DRGE pela técnica de hiatoplastia e "short floppy Nissen" pela via laparoscópica. O tratamento cirúrgico foi indicado baseado nos critérios estabelecidos pelo I Consenso Brasileiro de Doença do Refluxo Gastroesofágico.

No pré e pós-operatório (3 meses), além dos exames rotineiros de endoscopia digestiva alta e radiografia contrastada de esôfago, estômago e duodeno, foram realizadas sistematicamente eletromanometria esofágica e pHmetria de 24 horas, objetos deste estudo. Foram considerados critérios de exclusão: pacientes submetidos a cirurgia prévia em esôfago e estômago, complicações da DRGE (úlcera ou estenose esofágica e esôfago de Barrett), hérnia paraesofágica ou mista.

A eletromanometria esofágica foi realizada em todos os pacientes no Laboratório de Provas Funcionais do Aparelho Digestivo da Faculdade de Medicina do Triângulo Mineiro - FMTM, Uberaba, MG, pelo mesmo profissional, com suspensão prévia (por 7 dias) de medicamentos que pudessem alterar os resultados dos exames, como inibidores de bombas de prótons. Utilizaram-se para o exame sonda de polivinil (Sinetics gastro ${ }^{\circledR}$ ) com seis canais (quatro radiais) e a técnica de tração intermitente da mesma. Perfundindo-se água destilada, com auxílio de bomba de perfusão capilar-hidráulica $\left(\right.$ Biomedics $\left.^{\circledR}\right)$, os resultados eram registrados em um polígrafo (Polygram 4.21, Synetics Medical ${ }^{\circledR}$ ). Os parâmetros avaliados na manometria foram: localização, extensão, pressão média em repouso do EIE (expiratória final) e amplitude de pressão do corpo
esofágico.O resultado final de cada medida foi obtido através da média aritmética de, no mínimo, três valores do traçado.

Imediatamente após a eletromanometria esofágica procedeu-se à pHmetria ácida prolongada. A sonda de $2,1 \mathrm{~mm}$ de diâmetro era introduzida por via nasal com eletrodo de antimônio posicionado $5 \mathrm{~cm}$ acima do EIE. Após 24 horas, a sonda era retirada e os dados armazenados no dispositivo portátil, descarregados para sistema de computação previamente instalado (Esophagram ${ }^{\circledR}$ ).

O procedimento cirúrgico realizado foi a hiatoplastia com válvula total anti-refluxo(13) ("short floppy Nissen") por via laparoscópica. Iniciou-se a cirurgia através da abertura da "pars condensa" da membrana frenoesofágica, expondo-se o esôfago terminal e a transição esôfago gástrica. A hiatoplastia era realizada com pontos separados em X com fio inabsorvível. A válvula anti-refluxo total era feita através do envolvimento de um segmento de esôfago com a parede anterior e alta do fundo gástrico. Para tal, a parede anterior do estômago era passada posteriormente ao esôfago. Confirmado o posicionamento sem tensão do estômago, a válvula era confeccionada com três pontos separados, com fio inabsorvível (cerca de $3 \mathrm{~cm}$ ). Em nenhum caso foram seccionados os vasos gástricos curtos. Não houve necessidade de conversão nem complicações perioperatórias. No pós-operatório, a dieta líquida era instituída após 12 horas e os pacientes recebiam alta no final do primeiro dia.

O seguimento ambulatorial foi semanal no primeiro mês, quinzenal no segundo, mensal até o terceiro mês, quando se realizavam os exames de controle pós-operatório.

Analisaram-se estatisticamente os achados eletromanométricos e pHmétricos no pré e pós-operatório. Quando a distribuição da variável era normal (teste de Kolmogorov-Smirnov), utilizouse o teste $t$ de Student pareado. Quando a distribuição não foi considerada normal, aplicaram-se testes não-paramétricos (Wilcoxon, Mann-Whitney, Kruskal-Wallis). Padronizou-se como aceitação de erro $\alpha=0,05$.

$\mathrm{O}$ protocolo de pesquisa foi submetido e aprovado pelos Comitês de Ética em Pesquisa da FMTM e da Universidade Federal de São Paulo (UNIFESP), São Paulo, SP.

\section{RESULTADOS}

Quanto ao sexo, 18 (30,5\%) pacientes eram do sexo masculino, enquanto $41(69,5 \%)$ pertenciam ao sexo feminino. A idade média foi 43,8 anos (variação 21-75 anos) (Figura 1).

Através do exame eletromanométrico, o EIE localizou-se mais distalmente no pós-operatório $(42,3 \pm 2,6 \mathrm{~cm})$ em média quando comparado com o pré-operatório $(41,2 \pm 3,2 \mathrm{~cm})$, havendo significância estatística $(P=0,014)$. Sua extensão também aumentou, passando de $2,0 \mathrm{~cm}$ para $2,5 \mathrm{~cm}$ no pós-operatório, também com significância estatística $(P=0,0002)$. Houve, ainda, aumento significativo na pressão de repouso do EIE, cuja mediana elevou-se de $15,0 \mathrm{~mm} \mathrm{Hg}$ para $21,7 \mathrm{~mm} \mathrm{Hg}$ do pré para o pós-operatório $(P<0,001)$ (Figura 2, Tabela 1).

Quanto ao corpo esofágico detectou-se redução das amplitudes de pressão no pós-operatório $(70 \mathrm{~mm} \mathrm{Hg})$ quando comparado com os valores obtidos no pré-operatório $(78,0 \mathrm{~mm} \mathrm{Hg})$, valores estes estatisticamente significantes $(P=0,024)$. 


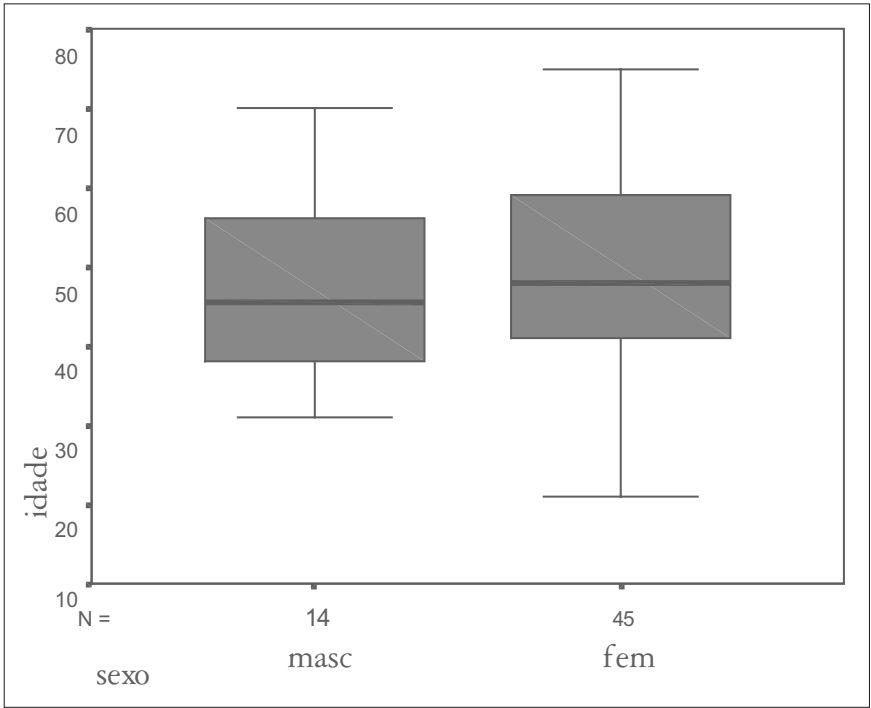

FIGURA 1 - Representação da distribuição dos 59 pacientes e a correlação entre as variáveis idade e sexo

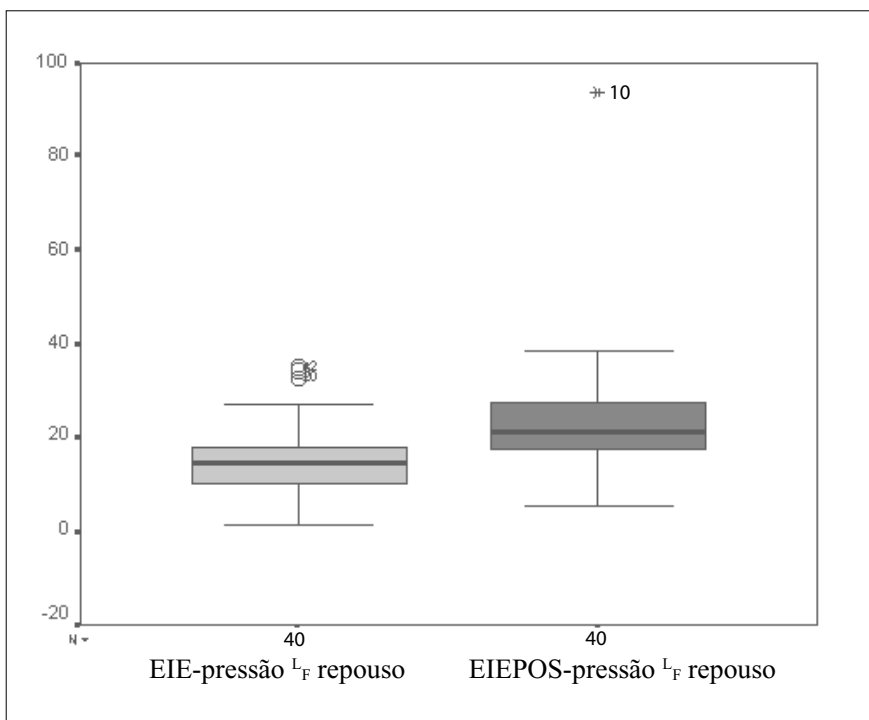

FIGURA 2 - Representação gráfica da diferença de pressão de repouso do esfíncter inferior do esôfago no pré e pós-operatório

TABELA 1 - Comparação dos valores obtidos através da eletromanometria esofágica nos períodos pré e pós-operatório da cirurgia de short floppy Nissen

\begin{tabular}{lccc}
\hline & Pré-operatório & Pós-operatório & $\boldsymbol{P}^{* *}$ \\
\hline Localização do EIE $(\mathrm{cm})$ & $41,2(3,2)^{*}$ & $42,3(2,6) *$ & 0,0136 \\
Extensão do EIE $(\mathrm{cm})$ & $2,0(1,0-4,0)$ & $2,5(1,0-6,0)$ & 0,0002 \\
Pressão de repouso do EIE $(\mathrm{mm} \mathrm{Hg})$ & $15,0(1,1-34,9)$ & $21,7(12,1-21,7)$ & $<0,001$ \\
Pressão do corpo esofágico $(\mathrm{mm} \mathrm{Hg})$ & $78,0(25,0-157,0)$ & $70,0(34,0-117,0)$ & 0,024 \\
\hline
\end{tabular}
normal (teste de Wilcoxon).
Através da pHmetria esofágica de 24 horas, constatou-se que houve melhora em todos os parâmetros. Inicialmente com redução significativa do número de refluxo ácidos totais no pós-operatório $(3,0)$, em relação ao pré-operatório $(68,0) P<0,001$. O número de refluxos ácidos prolongados $(>5$ minutos) foi maior do ponto de vista estatístico $(P=0,001)$ no pré-operatório $(2,0)$, quando comparado com os valores obtidos no pós-operatório $(0,0)$.

A mediana do número de refluxos ácidos prolongados com os pacientes em decúbito dorsal foi de $1,0(0,0-12,0)$ no préoperatório e de $0,0(0,0-8,0)$ no pós-operatório, $\operatorname{com} P<0,001$. O mesmo ocorreu com número de refluxos ácidos prolongados na posição ereta, variando de $0,0(0,0-22,0)$ no pré-operatório para $0,0(0,0-2,0)$ no pós-operatório, apresentando diferenças significantes com o tratamento estatístico empregado $(P=$ $0,001)$. A análise da fração de tempo de acidificação convertida em minutos, foi significantemente maior $(P<0,0001)$ no préoperatório (5,5 minutos), quando comparada com o pós-operatório (0,1 minuto). A análise de índice de DeMeester demonstrou redução significante $(P<0,001)$ quando se comparam os valores obtidos no pós-operatório $(33,0)$ com os obtidos no pré-operatório $(0,8)$ (Figura 3, Tabela 2).

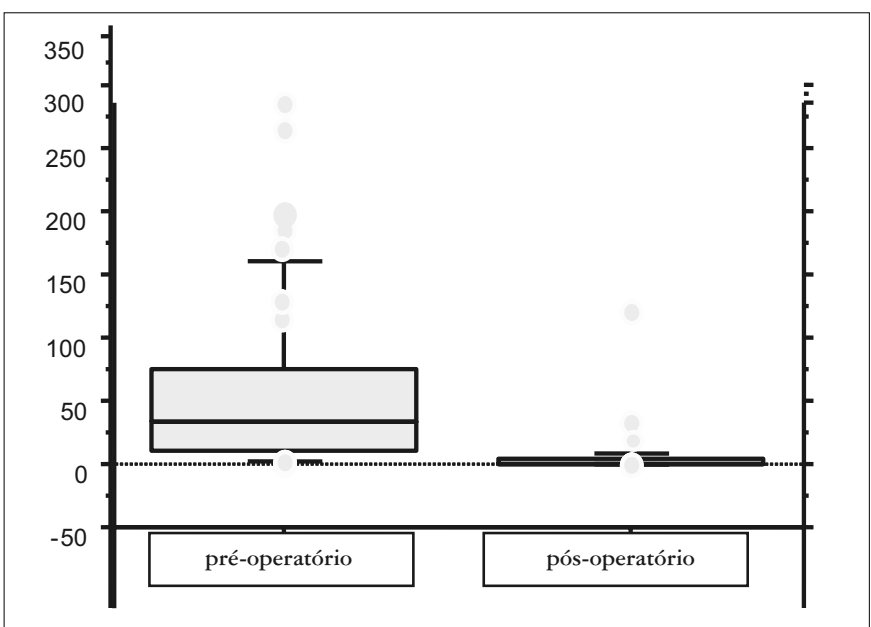

FIGURA 3 - Representação gráfica do índice de DeMeester nos períodos pré e pós-operatório

TABELA 2 - Comparação dos valores de mediana obtidos através da eletromanometria esofágica nos períodos pré e pós-operatório da cirurgia de short floppy Nissen

\begin{tabular}{lccc}
\hline & Pré-operatório & Pós-operatório & $\boldsymbol{P}^{*}$ \\
\hline Número total de refluxos & $68,0(0,0-898,0)$ & $3,0(0,0-162,0)$ & $<0,001$ \\
Número de refluxos prolongados & $2,0(0,0-25,0)$ & $0,0(0,0-8,0)$ & 0,0001 \\
$\begin{array}{l}\text { Número total de refluxos } \\
\text { prolongados deitado }\end{array}$ & $1,0(0,0-12,0)$ & $0,0(0,0-8,0)$ & $<0,001$ \\
$\begin{array}{l}\text { Número total de refluxos } \\
\text { prolongados em pé }\end{array}$ & $0,0(0,0-22,0)$ & $0,0(0,0-2,0)$ & 0,001 \\
$\begin{array}{l}\text { Tempo de acidificação } \\
\text { Índice de DeMeester }\end{array}$ & $5,5(0,0-146,0)$ & $0,1(0,0-28,6)$ & $<0,0001$ \\
\hline *Intervalo de confiança de 95\% & $33,0(0,3-327,0)$ & $0,8(0,2-121,5)$ & $<0,001$ \\
\hline
\end{tabular}




\section{DISCUSSÃO}

Este estudo é parte do projeto iniciado em 1997 sobre a cirurgia da transição esofagogástrica no Serviço de Cirurgia do Aparelho Digestivo da FMTM, aplicando a experiência do Serviço na realização de válvulas totais e no tratamento cirúrgico do megaesôfago por videolaparoscopia. Desde 1996 passou-se a empregar rotineiramente a eletromanometria e a pHmetria de 24 horas na avaliação do pré e pós-operatório de todos os pacientes.

Nesta casuística houve distribuição superior de pacientes no sexo feminino $(76,3 \%)$. Tal fato não corresponde ao comumente descrito na literatura. Parece que pacientes do sexo masculino apresentam mais refluxo gastroesofágico que a população semelhante do sexo feminino ${ }^{(20)}$, sendo que alguns autores chegam a propor o uso de padrões diferentes para cada sexo ${ }^{(13)}$. Com relação à idade, obteve-se valor médio de 43,8 anos em concordância à literatura, que verifica aumento da prevalência da DRGE com aumento da idade acima de 40 anos.

Estudos prévios demonstram bons resultados da cirurgia de válvula total para a correção da DRGE. DALLEMAGNE et al. ${ }^{(2)}$, por exemplo, com acompanhamento de 15 meses, obtiveram $90,5 \%$ de resultados satisfatórios.

Quanto à manometria, neste estudo ficou demonstrada elevação da pressão de repouso do EIE com a confecção da hiatoplastia e válvula total, passando de mediana de $15 \mathrm{~mm} \mathrm{Hg}$ para $21 \mathrm{~mm} \mathrm{Hg}$ no pós-operatório. A hipotonia esfincteriana está diretamente relacionada a maior predisposição ao refluxo gastroesofágico patológico ${ }^{(5)}$. Em experimentos com animais ${ }^{(16)}$ mostrou-se que o EIE normal, após miomectomia, chegou à medida próxima de zero de pressão, e rapidamente restauraram-se os valores normais após a fundoplicatura. A análise da localização do EIE no pós-operatório mostrou, nessa série, deslocamento caudal de $41,0 \mathrm{~cm}$ para $42,0 \mathrm{~cm}$. Observou-se ainda, aumento da extensão do EIE com a confecção da válvula anti-refluxo, passando de 2,0 para $2,5 \mathrm{~cm}$, semelhante ao que foi visto por LUNDELL et al. ${ }^{(11)}$, quando compararam as cirurgias de Rossetti (válvula total) e Toupet (parcial), observando elevação da extensão do esfíncter inferior do esôfago maior na válvula total do que na parcial e ambas com diferença significativa em relação ao pré-operatório. Com a extensão intra-abdominal da zona de alta pressão houve clara diferença entre o pré e pós-operatório, mesmo sem diferenças entre as técnicas. DeMEESTER et al. ${ }^{(4)}$, avaliando 100 pacientes submetidos a fundoplicatura à Nissen laparotômica, obtiveram o que foi chamado de extensão abdominal do EIE, significativamente diferente comparando pré $(1,5 \mathrm{~cm} \pm 0,9)$ e pós-operatório $(2,4 \mathrm{~cm}$ $\pm 1,1)$. Um dos objetivos da cirurgia para correção do refluxo ${ }^{(18)}$ é a obtenção deste segmento maior de esôfago em situação abdominal, submetendo-o dessa forma a pressão positiva. Na presente série a medida da distância do EIE à borda nasal, mostra que o objetivo em posicionar mais distalmente o EIE, foi atingido com a técnica empregada. Ainda quanto à manometria, houve redução dos valores de pressão no corpo esofágico no período pós-operatório passando de 78,0 para 70,0 mm Hg. Esta diminuição de pressão pode significar que o esôfago livre da esofagite diminuindo a hiper-reatividade, retornaria a seus níveis pressóricos próximos dos normais como supõem STEIN et al. ${ }^{(19)}$.
No presente estudo, quando se avaliou a pHmetria ácida de 24 horas, houve redução significante do número de refluxos ácidos totais no pós-operatório, quando comparados ao préoperatório, em concordância com os resultados apresentados por DeMEESTER e JOHNSON ${ }^{(3)}$. O número médio de refluxos obtido em pacientes no pré-operatório foi 63,5 , enquanto no pós-operatório foi 4,4. O grupo controle obteve média maior do que o grupo pós-operatório, ou seja, 20,6. Em 1995 PETERS et al. ${ }^{(16)}$ observaram também importante diminuição do número de refluxos ácidos nas 24 horas de pacientes com DRGE submetidos a fundoplicatura à Nissen por via aberta e laparoscópica. Não se encontrou nenhum estudo em que o número de refluxos ácido tenha aumentado no pós-operatório. Esses achados, assim como os da presente casuística, demonstram eficácia da hiatoplastia e fundoplicatura total ("short floppy Nissen") em corrigir o refluxo patológico, ao aproximar a níveis normais o número de refluxos ácidos. A análise da fração de tempo de acidificação neste estudo foi convertida em minutos, após correção do intervalo de tempo entre todos os exames, resultando em diferença significantemente maior no pré-operatório, quando comparada com o pós-operatório, ao mesmo tempo em que o índice de DeMeester apresentou redução significante no pós-operatório, quando se comparou ao valor obtido no pré-operatório. DeMEESTER e JOHNSON ${ }^{(3)}$, em 1975, obtiveram redução da fração de tempo de acidificação de $11,1 \%$ no pré-operatório para $0,2 \%$ no pós-operatório. Quanto ao índice de DeMeester, ele foi reduzido de 65,2 no pré-operatório para 6,7 no pós-operatório. PETERS et al. ${ }^{(16)}$, em 1995, obtiveram diminuição do tempo de acidificação de 14,8 para 0,42 minutos no pós-operatório de hiatoplastia e fundoplicatura à Nissen por laparoscopia. BLOMQVIST et al. ${ }^{(1)}$, em 1996, em ensaio comparando qualidade de vida após a fundoplicatura laparoscópica ou laparotômica, verificaram diminuição acentuada da fração de acidificação no pós-operatório dos dois grupos. No Brasil, FELIX et al. ${ }^{(6)} \mathrm{em} 2002$, observaram em seus pacientes queda dos índices de DeMeester de 16,7 no pré-operatório para 0,8 no pós-operatório.

Como se pode observar na Tabela 2 e Figura 3, três indivíduos ("outliers") mantiveram no pós-operatório o índice de DeMeester elevado, além de apresentarem diminuição da pressão do esfíncter inferior do esôfago. Quando avaliados através do exame radiográfico contrastado do esôfago, um deles apresentou migração precoce da válvula. Estes indivíduos devem ser interpretados como dentro do índice de falha terapêutica precoce ${ }^{(1,4,6,9)}$.

Deve-se lembrar que nesta série, o período de acompanhamento foi de 3 meses (pós-operatório), não sendo possível a avaliação da eficácia da cirurgia a longo prazo e nem de suas complicações tardias. De qualquer modo, admite-se que no intervalo de tempo avaliado, a hiatoplastia e a fundoplicatura foram eficientes em controlar ou curar a maioria dos doentes com DRGE.

Acredita-se que a manometria e pHmetria, a despeito de serem métodos de execução não tão simples, comparados à endoscopia digestiva alta, foram eficientes na avaliação de forma objetiva, os resultados da cirurgia. Destarte, como existe número apreciável de pacientes com DRGE e aspectos endoscópicos normais, esses exames poderiam ser empregados na sua correta avaliação pós-operatória. 


\section{CONCLUSÕES}

A eletromanometria esofágica e a pHmetria ácida prolongada demonstraram melhora em cada um dos seus parâmetros com significância estatística nos resultados observados, no período pré e pós-operatório de hiatoplastia e válvula anti-refluxo total, demonstrando a eficiência do procedimento operatório quando avaliado por esses exames, que permitem mensurar as características anatomofisiológicas do esôfago, aplicando-lhes valores numéricos objetivos de valor na prática médica diária, mas principalmente, na pesquisa clínica

Pastore R, Crema E, Silveira MC, Presoto AF, Herbella FAM, Del Grande JC. Electromanometry and acid pHmetry of 24 hours in postoperative evaluation of the hiatoplasty and total antireflux wrap laparoscopic. Arq Gastroenterol. 2006;43(2):112-6.

ABSTRACT - Background - The electromanometry and 24-hour esophageal pHmetry has been proposed as objective method in postoperative evaluation of antireflux surgery. Aim - Prospective evaluation of the results of cruroplasty and total fundoplication in the treatment of noncomplicated gastroesophageal reflux disease based on comparative results of pre and post operative manometry and pHmetry findings. Methods - Fifty-nine consecutive individuals with typical symptoms of gastroesophageal reflux disease were prospectively studied. All patients were submitted to a laparoscopic short floppy Nissen fundoplication between March, 2002 and August, 2003. All patients were submitted pre and postoperatively (3 months) to upper digestive endoscopy, manometry and pHmetry 24 hours. Results - Eighteen (30.5\%) patients were male and 41 (69.5\%) female, their average age was 43.8 years. There were differences comparing the pre and post operative period in the manometrical findings in the following items: localization of the lower esophageal sphincter $(41.2 \mathrm{~cm}$ and $42.3 \mathrm{~cm})$. Extension of the lower esophageal sphincter preoperative $(2.0 \mathrm{~cm}$ and $2.5 \mathrm{~cm})$, resting pressure of the lower esophageal sphincter $(15.0 \mathrm{~mm} \mathrm{Hg}$ and $21.5 \mathrm{~mm} \mathrm{Hg}$ ), and pressure of the esophageal body $(78.0 \mathrm{~mm} \mathrm{Hg}$ and $70.0 \mathrm{~mm} \mathrm{Hg})$. There were differences comparing the pre and post operative period in the pHmetrical findings in the following items: total number of acid refluxes (68.0 and 3.0), total number of acid refluxes more than 5 minutes (2.0 and 0.0 ), acid reflux more than 5 minutes in supine position ( 1.0 and 0.0$)$, acid reflux more than 5 minutes in upright position (0.0 and 0.0), acidification period (5.5 and $0.1 \mathrm{~min}$ ), DeMeester score (33.0 and 0.8$)$. Conclusions - The esophageal manometry and pHmetry findings were improved in the post operative period with statistical significance when compared to the pre operative period. The exams were considered efficient in the evaluation of the surgical procedure.

HEADINGS - Gastroesophageal reflux. Manometry. Hernia, hiatal. Fundoplication. Laparoscopy.

\section{REFERÊNCIAS BIBLIOGRÁFICAS}

1. Blomqvist A, Lönroth H, Dalenbäck J, Ruth M, Wiklund I, Lundell L. Quality of life assessment after laparoscopic and open fundoplications. Results of a prospective, clinical study. Scand J Gastroenterol. 1996;31:1052-8.

2. Dallemagne B, Taziaux P, Weerts J, Jehaes C, Markiewicz S. Chirurgie laparoscopique du reflux gastroesophagien. Ann Chir. 1994;48:159-64.

3. DeMeester TC, Johnson LF. Evaluation of the Nissen antireflux procedures by esophageal manometry and twenty-four hour pH monitoring. Am J Surg. 1975;29:94-100.

4. DeMeester TR, Bonavina L, Albertucci M. Nissen fundoplication for gastroesophageal reflux disease - evaluation of primary repair in 100 consecutive patients. Ann Surg. 1986;204:9-20.

5. Dodds WJ, Dent J, Hogan WJ, Helm JF, Hauser R, Patel GK, Egide MS. Mechanisms of gastroesophageal reflux in patients with reflux esophagitis. N Engl J Med. 1982;307:1547-52.

6. Felix VN, Yogi I, Perrini M, Echeverria R, Bernardi C. Surgical treatment of the non-complicated gastroesophageal reflux: fundoplication without division of the short gastric vessels. Arq Gastroenterol. 2002;39:93-7.

7. Gundmundsson K, Johnsson F, Joelsson B. The time pattern of gastroesophageal reflux. Scand J Gastroenterol. 1988;23:75.

8. Hinder RA, Libbey JS, Gorecki P, Bammer T. Antireflux surgery: indications, preoperative evaluation and outcome. Gastroenterol Clin North Am. 1999;28:987-1006.

9. Hogan WJ, Shaker R. Life after antireflux surgery (symposium). Am J Med. 2000;108(4A):181S-190S.

10. Kahrilas PJ. GERD revisted: advances in pathogenesis. Hepatogastroenterology. 1998;45:1301-7.

11. Lundell L, Abrahamsson H, Ruth M, Sandberg N, Olbe LC. Lower esophageal sphincter characteristics and esophageal acid exposure following partial or 360 degrees fundoplication: results of a prospective, randomized, clinical study. World J Surg. 1991;15:115-20.
12. Mittal RK, McCallum RW. Electrical and mechanical activity in the human lower esophageal sphincter on patients with reflux esophagitis. Gastroenterology. 1988;81:1182-9.

13. Mold JW, Reed LE, Davis AR, Allen ML, Robinson M. Prevalence of gastroesophageal reflux in elderly patients in a primary care setting. Am J Gastroenterol. 1991;86:965-70.

14. Nader F, Costa JSD, Nader GA, Motta GL. Prevalência de pirose em Pelotas, RS, Brasil: estudo de base populacional. Arq Gastroenterol. 2003;40:31-4.

15. Nasi A. Doença do refluxo gastroesofágico: reavaliação clínica, endoscópica e da monitorização do pH intraluminar esofágico [tese]. São Paulo: Faculdade de Medicina da Universidade de São Paulo; 1996.

16. Peters JH, Heimbucher J, Kauer WKH, Incarbone R, Bremner CG, DeMeester TR. Clinical and physiologic comparision of laparoscopic and open Nissen fundoplication. J Am Coll Surg. 1995; 180:385-93.

17. Richards KF, Fisher KS, Flores JH, Christensen BJ. Laparoscopic Nissen fundoplication cost, morbidity and outcome compared with open surgery. Surg Laparosc Endosc. 1996;6:140-3.

18. Spechler SJ. Gastroesophageal reflux study group. Comparision of medical and surgical therapy for complicated gastroesophageal reflux disease in veterans. N Engl J Med.1992;326:786-92.

19. Stein HJ, Bremner RM, Jamieson J, DeMeester TR. Effect of Nissen fundoplication on esophageal motor function. In: Paula AL, Hashiba K, Bafutto M, editores. Cirurgia videolaparoscópica. Goiânia; 1994. p.35-8.

20. Ter RB, Johnston BT, Castel DO. Influence of age and gender on gastroesophageal reflux in symptomatic patient. Dis Esophagus. 1998;11:106-8. 